\title{
Intrinsic Rates and Activation Free Energies from Single-Molecule Pulling Experiments
}

\author{
Olga K. Dudko, ${ }^{1}$ Gerhard Hummer, ${ }^{2}$ and Attila Szabo ${ }^{2}$ \\ ${ }^{1}$ Mathematical and Statistical Computing Laboratory, Division of Computational Bioscience, Center for Information Technology, \\ National Institutes of Health, Bethesda, Maryland 20892, USA \\ ${ }^{2}$ Laboratory of Chemical Physics, National Institute of Diabetes and Digestive and Kidney Diseases, National Institutes of Health, \\ Bethesda, Maryland 20892-0520, USA
}

(Received 29 November 2005; published 15 March 2006)

\begin{abstract}
We present a unified framework for extracting kinetic information from single-molecule pulling experiments at constant force or constant pulling speed. Our procedure provides estimates of not only (i) the intrinsic rate coefficient and (ii) the location of the transition state but also (iii) the free energy of activation. By analyzing simulated data, we show that the resulting rates of force-induced rupture are significantly more reliable than those obtained by the widely used approach based on Bell's formula. We consider the uniqueness of the extracted kinetic information and suggest guidelines to avoid overinterpretation of experiments.
\end{abstract}

DOI: 10.1103/PhysRevLett.96.108101

PACS numbers: 87.15.Aa

How should single-molecule pulling experiments be analyzed to extract reliable information about the kinetics of molecular transitions such as unfolding or ligand dissociation in the absence of external forces? Unlike the rigorous formalisms developed for thermodynamic properties $[1,2]$, rate estimates remain contentious. Even under the assumption that the pulling direction alone is a good reaction coordinate, which is not the case in general [35], there are at least three competing theories. At the simplest level, the phenomenological theory [6] assumes that the rate of rupture $k(F)$ scales with the exponential of the applied force $F$ according to Bell's formula [7]: $k(F)=$ $k_{0} e^{F x^{\ddagger}}$ (throughout the Letter, we measure energy in units of $k_{B} T$, with $k_{B}$ being Boltzmann's constant and $T$ the absolute temperature). This approach is widely used to extract from experiment the intrinsic rate coefficient $\left(k_{0}\right)$ and the distance along the pulling direction between the free-energy minimum and the transition state $\left(x^{\ddagger}\right)$. For experiments at constant pulling speed $V$ where the applied force grows linearly with time $t$ as $F(t)=K V t$ (with $K$ the effective spring constant and $K V$ the force loading rate), the mean rupture force is predicted to grow proportionally to the logarithm of the pulling speed [8,9], $\langle F\rangle \sim \ln V$.

Recently, Hummer and Szabo [10] proposed a more sophisticated but still analytically tractable procedure. By applying the Kramers theory of diffusive barrier crossing to a simple model free-energy surface, one can extract not only $k_{0}$ and $x^{\ddagger}$ but also $\Delta G^{\ddagger}$, the free energy of activation in the absence of external forces. Their theory reduces to the phenomenological approach when $\Delta G^{\ddagger} \rightarrow \infty$ and predicts that, at intermediate pulling speeds, $\langle F\rangle \sim(\ln V)^{1 / 2}$. At the same time, Dudko et al. [11], using the Kramers theory in conjunction with certain scaling laws obtained by Garg [12] for high forces, showed how to extract the critical force $F_{c}$ at which the barrier to rupture vanishes, the apparent free energy of activation, and a parameter proportional to the diffusion constant. Their theory predicts that $\langle F\rangle \sim(\ln V)^{2 / 3}$. Thus, these two theories disagree not only with the phenomenological approach but also with each other. One may suspect that these theories are valid in different regimes and produce highly model-dependent results when applied to experiments. Here we clarify this situation by first casting all these theories into a common framework and then proposing a unified formalism for extracting $k_{0}, x^{\ddagger}$, and $\Delta G^{\ddagger}$ from pulling experiments.

During an irreversible molecular transition under the influence of a force $F$ exerted by a soft pulling spring, the molecule moves on a combined free-energy surface $U(x)=U_{0}(x)-F x$ along the pulling direction $x$. The bare free energy $U_{0}(x)$ is assumed to have a single well at $x=0$ and a barrier of height $\Delta G^{\ddagger}$ at $x=x^{\ddagger}$ [Fig. 1(a)]. Under the application of the external force $F$, the barrier height $\Delta U(F)$ of the combined surface $U(x)$ decreases [Fig. 1(b)]. Although other experiments have been proposed [13], we focus here on experiments at constant force and constant pulling speed where the time to rupture and the force at rupture, respectively, are measured. Since the escape process is stochastic, both the waiting times and rupture forces
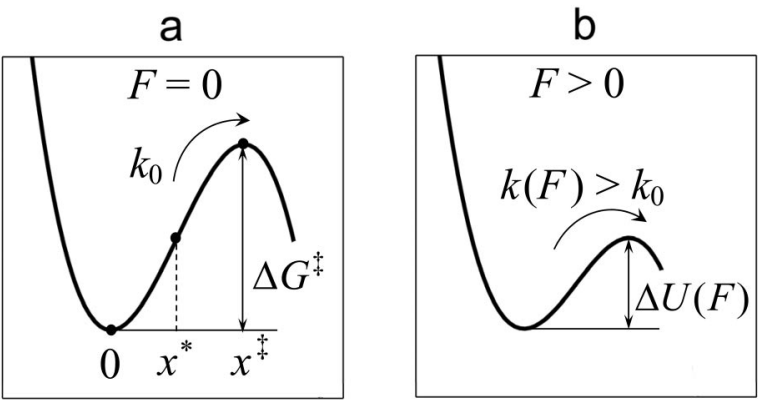

FIG. 1. Schematic representation of a single-well free-energy surface. (a) Intrinsic free-energy surface $U_{0}(x)$ with minimumto-barrier distance $x^{\ddagger}$, activation free energy $\Delta G^{\ddagger}$, and inflection point $x^{*}$; (b) decrease in the free-energy barrier $\Delta U(F)$ of the combined free-energy surface, $U(x)=U_{0}(x)-F x$, with increasing external force $F$. 
differ from one experiment to another, resulting in distributions $p(t)$ and $p(F)$ of rupture times and forces.

For irreversible rupture under the influence of an external force $F(t)$ that increases monotonically with time $t$ (as in a constant-speed experiment), the distribution of rupture forces is related to the survival probability $S(t)$ (the probability that the system is still intact at time $t$ ) by $p(F) d F=$ $-\dot{S}(t) d t$, where $\dot{X} \equiv d X / d t$. The survival probability is assumed to satisfy a first-order rate equation with a timedependent rate coefficient

$$
\dot{S}(t)=-k(F(t)) S(t) .
$$

At extreme pulling speeds or external forces, the adiabatic approximation underlying Eq. (1) breaks down [10] and the following theory becomes inapplicable. It follows from Eq. (1) that the distribution of rupture forces is

$$
p(F)=\frac{k(F)}{\dot{F}} e^{-\int_{0}^{F}\left[k\left(F^{\prime}\right) / \dot{F}\right] d F^{\prime}} .
$$

The mean rupture force is then $\langle F\rangle=\int F p(F) d F$ and its variance is $\sigma_{F}^{2}=\left\langle F^{2}\right\rangle-\langle F\rangle^{2}$.

Assume that along the pulling coordinate $x$ the combined free-energy surface is given by $U(x)=U_{0}(x)+$ $K(x-V t)^{2} / 2$. For a soft effective spring, $K \ll$ $2 \Delta G^{\ddagger} / x^{\ddagger 2}$, this can be approximated as $U(x) \approx U_{0}(x)-$ $F(t) x$, with $F(t)=K V t$. One way to obtain the rate of escape is to use Bell's expression, as is done in the phenomenological approach. Alternatively $[8,14]$, one can specify the free-energy surface $U_{0}(x)$ and use the Kramers theory [15], which, for sufficiently high barriers, predicts the rate $k$ in the presence of a force $F$ to be $k / k_{0}=\left[\int_{\text {well }} e^{-U_{0}(x)+F x} d x \int_{\text {barrier }} e^{U_{0}(x)-F x} d x\right]^{-1} \times$ $\int_{\text {well }} e^{-U_{0}(x)} d x \int_{\text {barrier }} e^{U_{0}(x)} d x$, where the integrals extend over the well and barrier regions, respectively. There are at least two single-well model free-energy surfaces $U_{0}(x)$ for which the integral in Eq. (2) can be evaluated analytically for external forces that grow linearly with time: the cusp surface [10], $U_{0}(x)=\Delta G^{\ddagger}\left(x / x^{\ddagger}\right)^{2}$ for $\left(x<x^{\ddagger}\right)$ and $-\infty$ for $\left(x \geq x^{\ddagger}\right)$, and the linear-cubic surface, $U_{0}(x)=$ $(3 / 2) \Delta G^{\ddagger} x / x^{\ddagger}-2 \Delta G^{\ddagger}\left(x / x^{\ddagger}\right)^{3}$. Using the above formalism, we find that the escape rate for both these surfaces and for the phenomenological theory can be written in a unified form:

$$
k(F)=k_{0}\left(1-\frac{\nu F x^{\ddagger}}{\Delta G^{\ddagger}}\right)^{1 / \nu-1} e^{\Delta G^{\ddagger}\left[1-\left(1-\nu F x^{\ddagger} / \Delta G^{\ddagger}\right)^{1 / \nu}\right]},
$$

where $\nu=2 / 3$ and $1 / 2$ correspond to the linear-cubic and cusp free-energy surfaces, respectively. For $\nu=1$, and for $\Delta G^{\ddagger} \rightarrow \infty$ independent of $\nu$, the expression reduces to the phenomenological result. In the limit $F \rightarrow 0$, $\ln \left(k(F) / k_{0}\right)=F x^{\ddagger}\left(1+(\nu-1) / \Delta G^{\ddagger}\right)$. When $\nu \neq 1$, permissible values of the force $F$ in Eq. (3) are limited from above by the value of the critical force $F_{c}=\Delta G^{\ddagger} / \nu x^{\ddagger}$ at which the barrier disappears. Because Eq. (3) is based on the Kramers theory in the high-barrier limit, it incorrectly predicts that $k\left(F_{c}\right)=0$ rather than $k\left(F_{c}\right) \gg k_{0}$. This could be rectified by using more accurate expressions based on the theory of first passage times [16], but then the integral in Eq. (2) could not be evaluated analytically. The escape rate in Eq. (3) describes both constant-force and timedependent linear force experiments.

When $F(t)=K V t$, the distribution of rupture forces can be obtained from Eqs. (2) and (3) analytically as

$$
\begin{aligned}
p(F \mid V)= & (K V)^{-1} k(F) e^{k_{0} / x^{\ddagger} K V} \\
& \times e^{-\left[k(F) / x^{\ddagger} K V\right]\left[1-\left(\nu F x^{\ddagger} / \Delta G^{\ddagger}\right)\right]^{1-1 / \nu},}
\end{aligned}
$$

and the asymptotic expressions for the mean rupture force and variance are [12]

$$
\begin{gathered}
\langle F\rangle \cong \frac{\Delta G^{\ddagger}}{\nu x^{\ddagger}}\left\{1-\left[\frac{1}{\Delta G^{\ddagger}} \ln \frac{k_{0} e^{\Delta G^{\ddagger}+\gamma}}{x^{\ddagger} K V}\right]^{\nu}\right\}, \\
\sigma_{F}^{2} \cong \frac{\pi^{2}}{6 x^{\ddagger^{2}}}\left[\frac{1}{\Delta G^{\ddagger}} \ln \frac{k_{0} e^{\Delta G^{\ddagger}+\tilde{\gamma}}}{x^{\ddagger} K V}\right]^{2 \nu-2} .
\end{gathered}
$$

Here $\tilde{\gamma}=\gamma^{2}-3 / \pi^{2} \psi^{\prime \prime}(1) \approx 1.064$, where $\gamma=0.577 \ldots$ is the Euler-Mascheroni constant and $\psi^{\prime \prime}(1)=-2.404 \ldots$ is a particular value of the tetragamma function [17]. When $\gamma$ is formally set to zero, Eq. (5) gives the maximum (mode) of the rupture-force distribution to a good approximation.

The variance $\sigma_{F}^{2}$ in Eq. (6) for the phenomenological theory $(\nu=1)$ is independent of the speed $V$. Thus, if the experimental variance depends on $V$, the phenomenological approach is invalid even if it reasonably describes the available data for the mean or maximum rupture force. Since $\langle F\rangle \sim(\ln V)^{\nu}$, it is tempting to analytically continue Eqs. (3)-(6) to all $\nu$ and use $\nu$ as an additional fitting parameter.

So far, our results appear to depend strongly on the model (cusp vs linear-cubic free-energy surface). We now show that the results based on the linear-cubic freeenergy surface are less model-dependent than it appears at first sight. The reason is that for high forces (but with barriers still sufficiently high so that the Kramers theory is still applicable), all smooth combined free-energy surfaces can be well represented by a cubic polynomial. This idea had been first exploited in the context of Josephson junctions by Kurkijärvi [18] and further developed by Garg [12]. In the context of the single-molecule pulling experiments, this formalism was first used by Dudko et al. [11].

To rederive the results for the linear-cubic free-energy surface from a more general point of view, we first expand the combined free-energy surface around the inflection point $x^{*}, U^{\prime \prime}\left(x^{*}(F)\right)=0$ [Fig. 1(a)], to cubic order: $U(x) \approx$ $U_{0}\left(x^{*}\right)+\left(x-x^{*}\right) U_{0}^{\prime}\left(x^{*}\right)+\left(x-x^{*}\right)^{3} U_{0}^{\prime \prime \prime}\left(x^{*}\right) / 6-F x$. When the free-energy surface has a sharp barrier (the cusp surface being an extreme case), the range of validity of this expansion is rather limited. The extrema of this truncated freeenergy surface are found by solving $U^{\prime}\left(x_{ \pm}(F)\right)=0$. Then the minimum-to-barrier distance is $\Delta x(F)=x_{+}-x_{-}$, and the barrier height is $\Delta U(F)=U\left(x_{+}\right)-U\left(x_{-}\right)$. We now define the apparent minimum-to-barrier distance and the 
apparent barrier height by taking the formal limit $F \rightarrow 0$ : $x_{c}^{\ddagger} \equiv \Delta x(0), \quad \Delta G_{c}^{\ddagger} \equiv \Delta U(0)$. These apparent parameters are given by the first and the third derivative of the free-energy surface at the inflection point as $x_{c}^{\ddagger}=$ $2\left[2 U_{0}^{\prime}\left(x^{*}\right)\right]^{1 / 2} /\left[-U_{0}^{\prime \prime \prime}\left(x^{*}\right)\right]^{1 / 2}, \Delta G_{c}^{\ddagger}=(2 / 3)\left[2 U_{0}^{\prime}\left(x^{*}\right)\right]^{3 / 2} /$ $\left[-U_{0}^{\prime \prime \prime}\left(x^{*}\right)\right]^{1 / 2}$. Applying the Kramers theory to the above truncated free-energy surface leads to the escape rate given by Eq. (3) for $\nu=2 / 3$ with substitutions $\Delta G^{\ddagger} \rightarrow \Delta G_{c}^{\ddagger}$, $x^{\ddagger} \rightarrow x_{c}^{\ddagger}$. For $F(t)=K V t$, the distribution of rupture forces, the mean rupture force and its variance are given by Eqs. (4)-(6) with $\nu=2 / 3$ and the substitutions $\Delta G^{\ddagger} \rightarrow$ $\Delta G_{c}^{\ddagger}, x^{\ddagger} \rightarrow x_{c}^{\ddagger}$.

In Fig. 2, we compare the analytical results in Eqs. (4)(6) with $\nu=2 / 3$ to Brownian dynamics simulations for force-induced rupture of a system described by the linearcubic free-energy surface. The external force was ramped up linearly with time. At pulling speeds where rupture occurs below the critical force $F_{c}$, the formulas are found to be quite accurate; at high pulling speeds, they break down as pointed out above.

Even for this simple model potential, the mean rupture force is a nonlinear function of the logarithm of the loading rate. Nevertheless, at low to intermediate speeds, a linear fit of the phenomenological model $(\nu=1)$ to the simulated $\langle F\rangle$ is quite good (Fig. 2). Within the framework of this widely used procedure, one might incorrectly attribute the

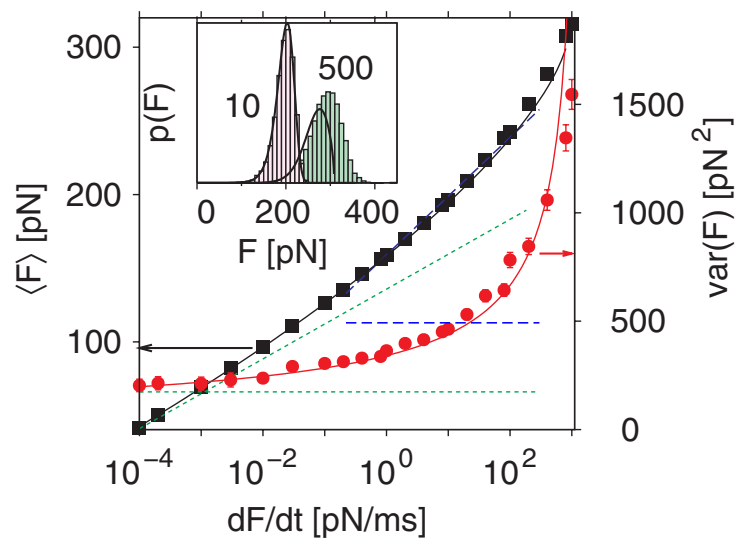

FIG. 2 (color online). Comparison of analytical results [solid lines; $\nu=2 / 3$ in Eqs. (5) and (6)] and Brownian dynamics simulations (symbols) for the mean (black squares) and variance (red circles) of the rupture force for the linear-cubic free-energy surface with $x^{\ddagger}=0.4 \mathrm{~nm}, \Delta G^{\ddagger}=20 k_{B} T$, and the diffusion coefficient $D$ chosen so that the Kramers rate $k_{0}=3 \pi^{-1} \times$ $\Delta G^{\ddagger}\left(x^{\ddagger}\right)^{-2} D \exp \left(-\Delta G^{\ddagger}\right)=10^{-4} \mathrm{~s}^{-1}$. Short-dashed (green) lines are the predictions of the phenomenological theory with the above parameters. Long-dashed (blue) lines are the fit of the phenomenological model $(\nu=1)$ to $\langle F\rangle$ for loading rates from 0.4 to $100 \mathrm{pN} / \mathrm{ms}$. Even though the fit to $\langle F\rangle$ is quite good, $k_{0}$ is too large (by a factor of 33). The inset compares the distributions of the rupture force from theory Eq. (4) (lines) and simulations (shaded bars) for loading rates of 10 and $500 \mathrm{pN} / \mathrm{ms}$. Although Eq. (4) already fails at $500 \mathrm{pN} / \mathrm{ms}$, the range of validity of Eqs. (5) and (6) for $\langle F\rangle$ and $\sigma_{F}^{2}$ extends further. curvature at higher force loading rates to some other physical process, such as a switch from one dominant freeenergy barrier to another. However, the dependence of the variance $\sigma_{F}^{2}$ on the loading rate immediately shows that the phenomenological model is inadequate here, incorrectly predicting $\sigma_{F}^{2}$ to be constant. Thus, if the experimental variance depends on the loading rate, one should view the parameters $k_{0}$ and $x^{\ddagger}$ extracted from the linear fit to the mean (or maximum) of the rupture-force distribution as suspect. For the parameters used in Fig. 2, the phenomenological rate $k_{0}$ is too high (by a factor of 33 ) and $x^{\ddagger}$ is too small (by a factor of 0.59). For smaller $\Delta G^{\ddagger}$, the errors are expected to be even greater since the phenomenological theory corresponds to the $\Delta G^{\ddagger} \rightarrow \infty$ limit.

As recently noted by Raible et al. [19], if the adiabatic approximation, Eq. (1), is valid for experiments at constant pulling speed $V$, the product of $V$ and the logarithm of the survival probability should be independent of $V$. By extending this observation, we can establish the relation between constant-force experiments [measuring $k(F)$ ] and constant-speed experiments [measuring $p(F \mid V)$ ]. For $F(t)=K V t$, it can be shown from Eq. (1) that

$$
k(F)=\frac{K V p(F \mid V)}{1-\int_{0}^{F} p\left(F^{\prime} \mid V\right) d F^{\prime}} \quad \forall V .
$$

As a consistency check, we show in Fig. 3 that Eq. (7) indeed collapses the rupture-force histograms from simulations at pulling speeds covering 7 orders of magnitude onto a single master curve for $k(F)$ given by the Kramers approximation, Eq. (3).

We have seen that the $\nu=2 / 3$ results for the linearcubic free-energy surface and for any smooth surface ex-

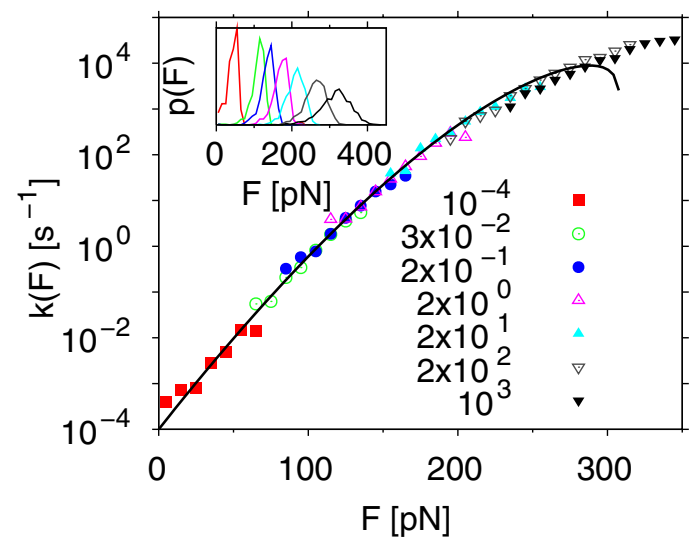

FIG. 3 (color online). Constant-force rupture rate $k(F)$ from collapse of constant-speed rupture-force histograms using Eq. (7). Simulation data (symbols) cover pulling speeds $V$ from $10^{-4}$ to $10^{3} \mathrm{pN} / \mathrm{ms}$, as indicated (see Fig. 2 for details of simulations), with each $V$ probing a different range of $F$. The solid line is the analytical prediction for $k(F)$, Eq. (3), with $\nu=$ $2 / 3$. Deviations at the highest pulling speeds (and rupture forces) are caused by a breakdown of the Kramers high-barrier approximation. The inset shows the force histograms (lowest to highest ramp speed from left to right) that were used in Eq. (7). 
TABLE I. Model parameters for unfolding of titin and RNA obtained by fitting high-force $(\nu=2 / 3)$ and phenomenological theories $(\nu=1)$ to mean-force data generated for the cusp model $(\nu=1 / 2)$.

\begin{tabular}{lcccccc}
\hline \hline & & Titin & & \multicolumn{2}{c}{ RNA } & \\
& $\nu=1 / 2$ & $\nu=2 / 3$ & $\nu=1$ & $\nu=1 / 2$ & $\nu=2 / 3$ & $\nu=1$ \\
\hline$k_{0}\left[10^{-4} \mathrm{~s}^{-1}\right]$ & 1 & 2.4 & 38 & 1 & 1.1 & 34.3 \\
$x_{c}^{\ddagger}[\mathrm{nm}]$ & 0.4 & 0.34 & 0.21 & 12 & 11.8 & 9.7 \\
$\Delta G_{c}^{\ddagger}\left[k_{B} T\right]$ & 20 & 17.6 & $\cdots$ & 40 & 31.3 & $\cdots$ \\
\hline \hline
\end{tabular}

panded around its inflection point are identical when one introduces apparent $\Delta G_{c}^{\ddagger}$ and $x_{c}^{\ddagger}$. How reasonable are these apparent quantities for other surfaces? For a quartic freeenergy surface, $U_{0}=2 \Delta G^{\ddagger}\left(x / x^{\ddagger}\right)^{2}-\Delta G^{\ddagger}\left(x / x^{\ddagger}\right)^{4}$, it can be shown that $x_{c}^{\ddagger}=(2 \sqrt{2} / 3) x^{\ddagger}$ and $\Delta G_{c}^{\ddagger}=4(2 / 3)^{7 / 2} \Delta G^{\ddagger}$. Both these coefficients are close to unity, and real and apparent parameters are essentially the same. An interesting example [11] is the Morse potential, $U_{0}=U_{\text {eq }}\{[1-$ $\left.\left.\exp \left(-2 b\left(x-R_{c}\right) / R_{c}\right)^{2}-1\right]\right\}$, which in the absence of force does not have a barrier ( $x^{\ddagger}$ is formally infinite). Nevertheless, by evaluating the first and third derivatives at the inflection point, one obtains an apparent free energy of activation, $\Delta G_{c}^{\ddagger}=2 U_{\mathrm{eq}} / 3$, and a distance to the transition state, $x_{c}^{\ddagger}=R_{c} / b$. Even though these parameters are not unreasonable qualitative characterizations of the potential, it is important to realize that they are only apparent.

To study the uniqueness of the extracted parameters, we use the high-force $(\nu=2 / 3)$ and phenomenological $(\nu=$ 1) theories to analyze data generated for the cusp surface $(\nu=1 / 2)$ with parameters that describe the unfolding of the protein titin $[10,20]$ and of RNA [1,21]. As shown in Table I, the two microscopic theories produce reasonably consistent transition state locations and rates, while rates from the phenomenological theory can be off by more than an order of magnitude.

For the reliable analysis of experiments in the regime where the barrier is high and the intrinsic rate is slow, the high-force theory $(\nu=2 / 3)$ should be globally fit to data for different pulling rates and/or from constant-force measurements. Fitting the mean rupture forces is insufficient to determine the three parameters $\left(k_{0}, x^{\ddagger}\right.$, and $\left.\Delta G^{\ddagger}\right)$. Including variances is helpful [10], but ideally the complete force (or rupture time) distribution should be used [22]. Bayesian (rather than least squares) fitting procedures are preferable here because they do not require binning of forces and automatically provide the range of acceptable parameters. To check for uniqueness, the data should also be fit using the $\nu=1 / 2$ theory (or with $\nu$ as a fitting parameter), and the rates, activation free energies, and transition state locations compared. For forces $F$ near $F_{c}$, Brownian dynamics simulations should be used instead of Eqs. (3)-(6). Since all these theories reduce to the $\nu=1$ theory when $\Delta G^{\ddagger} \rightarrow \infty$, a fit to the phenomenological model is unnecessary. Our illustrative calculations show that the two microscopic theories produce consistent rates and transition state locations (with somewhat larger differences in activation free energies), but the widely used phenomenological theory can be off substantially (more than an order of magnitude for the rate coefficient).

This research was supported by the Intramural Programs of the NIDDK and CIT, NIH.

[1] J. Liphardt, S. Dumont, S. B. Smith, I. Tinoco, Jr., and C. Bustamante, Science 296, 1832 (2002).

[2] G. Hummer and A. Szabo, Proc. Natl. Acad. Sci. U.S.A. 98, 3658 (2001).

[3] V. Barsegov and D. Thirumalai, Proc. Natl. Acad. Sci. U.S.A. 102, 1835 (2005).

[4] P. M. Williams, S. B. Fowler, R. B. Best, J. L. TocaHerrera, K. A. Scott, A. Steward, and J. Clarke, Nature (London) 422, 446 (2003).

[5] S. Kirmizialtin, L. Huang, and D. E. Makarov, J. Chem. Phys. 122, 234915 (2005).

[6] E. Evans, D. Berk, and A. Leung, Biophys. J. 59, 838 (1991).

[7] G. I. Bell, Science 200, 618 (1978).

[8] E. Evans and K. Ritchie, Biophys. J. 72, 1541 (1997).

[9] S. Izrailev, S. Stepaniants, M. Balsera, Y. Oono, and K. Schulten, Biophys. J. 72, 1568 (1997).

[10] G. Hummer and A. Szabo, Biophys. J. 85, 5 (2003).

[11] O. K. Dudko, A. E. Filippov, J. Klafter, and M. Urbakh, Proc. Natl. Acad. Sci. U.S.A. 100, 11378 (2003).

[12] A. Garg, Phys. Rev. B 51, 15592 (1995).

[13] V. Barsegov and D. Thirumalai, Phys. Rev. Lett. 95, 168302 (2005).

[14] B.E. Shapiro and H. Qian, Biophys. Chem. 67, 211 (1997).

[15] H. A. Kramers, Physica (Utrecht) 7, 284 (1940).

[16] A. Szabo, K. Schulten, and Z. Schulten, J. Chem. Phys. 72, 4350 (1980).

[17] M. Abramowitz and I. A. Stegun, Handbook of Mathematical Functions (Dover, New York, 1972).

[18] J. Kurkijarvi, Phys. Rev. B 6, 832 (1972).

[19] M. Raible, M. Evstigneev, P. Reimann, F. W. Bartels, and P. Ros, J. Biotechnol. 112, 13 (2004).

[20] M. Carrion-Vazquez, A. F. Oberhauser, S. B. Fowler, P. E. Marszalek, S. E. Broedel, J. Clarke, and J. M. Fernandez, Proc. Natl. Acad. Sci. U.S.A. 96, 3694 (1999).

[21] G. Hummer and A. Szabo, Acc. Chem. Res. 38, 504 (2005).

[22] J. Mathe, H. Visram, V. Viasnoff, Y. Rabin, and A. Meller, Biophys. J. 87, 3205 (2004). 